\title{
Residual Vibration of a Rotating Flexible Beam Subject to Prescribed Motion
}

\author{
K. Alnefaie, H. Diken and A. Alghamdi \\ Mechanical Engineering Department, King Abdulaziz University, \\ P. O. Box 80248, Jeddah 21589, Saudi Arabia \\ kalnefaie@kau.edu.sa
}

\begin{abstract}
In this paper, residual vibration of a servomotor driven rotating flexible beam is studied. The beam is modeled as an EulerBernoulli beam; it is rotated by a servomotor using triangular velocity profile (bang-bang trajectory). Analytical solution is also obtained by using Fourier series expansion of the acceleration of the rotating beam. Residual vibration amplitudes depend on the beam tip position at the end of the rotation, which is the function of rise time (the time to complete the rotation). It is found that if the rise time is the odd multiple of the beam period $1,3,5 \ldots$, residual vibration amplitudes are maximized. Residual vibration amplitude spectrum shows that for rise time to period ratios from 1.5 to 2.5 , residual vibration amplitudes are lowered to less than $3 \%$ of the maximum residual vibration amplitude, obtained for rise time, equal to the first natural period of the beam.
\end{abstract}

Keywords: Residual vibration, Servomotor, Rotating beam.

\section{Introduction}

Demand for high performance robotic systems quantified by high speed operation, high end-position accuracy and lower energy consumption has triggered a vigorous research in various multi-disciplinary areas, such as design and control of lightweight flexible robot arm. Flexible manipulators, although having some inherent advantageous over conventional rigid robots, have posed more stringent requirements on the control system design, such as accurate end point sensing and fast suppression of transient vibration during rapid arm movements. 
Point to point position control of a flexible beam is studied analytically and experimentally $\mathrm{in}^{[1,2]}$. Equations for a rotating Timoshenko beam are developed for pinned-free and clamped-free boundary conditions $\mathrm{in}^{[3]}$. Dynamic modeling and optimal control of a rotating Euler-Bernoulli beam is studied in ${ }^{[4]}$. Main objective of the paper was to control the vibration through force feedback control. Condition of a slewing beam using high speed camera system is studied in ${ }^{[5]}$. Results show that the natural frequency of the rotating beam is between the natural frequencies of fixed-free and free-free beam. Among many ${ }^{[6-13]}$ are also worth to mention which are related to a rotating flexible beam. A residual vibration spectrum for a rotating flexible beam is studied in $^{[14]}$. In this study cycloidal rise function is used to rotate the beam. Closed loop solution is obtained. Results show that, for frequency ratios of 2, 3, 4 ... residual vibration amplitudes became zero.

Shina and Brennan ${ }^{[15]}$ considered two methods for controlling the residual vibrations of a translating or rotating Euler-Bernoulli cantilever beam. Although a beam has an infinite number of vibration modes, when it simply changes its position by translation or rotation the first mode is the main contributor to the total response. Thus, the problem can be reduced to the base acceleration excitation of a single-degree-of-freedom system. Two simple methods are suggested for suppressing the residual vibration of such a system without considering any control algorithms.

Input shaping is also a control method that allows much higher speeds of motion by limiting vibration induced by the reference command. Vaughan, et al. ${ }^{[16]}$ analyze the compromise between rapidity of motion and shaper robustness for several input-shaping methods. Sorensen and Singhose ${ }^{[17]}$ also studied methods whereby arbitrary reference commands may be interpreted as input-shaped commands.

In this study, servomotor driven flexible beam is considered. Triangular velocity profile (bang-bang trajectory) is used to rotate the beam. Angular acceleration of the beam is approximated by Fourier series and analytic solution is obtained. Residual vibration amplitudes depend on the ratio of rise time to the beam vibration period. For ratios of $1,3,5, \ldots$ residual vibration amplitudes are maximized. It is possible to minimize residual vibration by choosing appropriate ratio of rise time to beam vibration period. 


\section{Formulation}

\subsection{Equation of a Rotating Beam}

Figure 1 shows a rotating flexible beam. Beam is considered as fixed-free Euler-Bernoulli beam. OXY is an inertial frame, Oxy is a rotating frame attached to the shaft. $\theta$ is a rotation angle of the shaft, $\mathrm{y}$ is the beam deflection and $m_{b}$ is the unit mass of the beam per length. The position vector of $m_{b}$ with respect to the rotating coordinates is

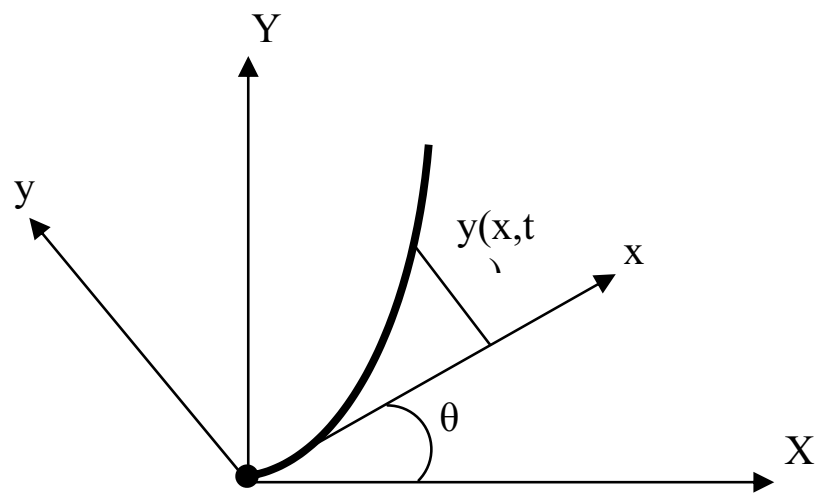

Fig. 1. Rotating flexible beam model.

$$
\vec{r}=x \vec{i}+y \vec{j}
$$

If $\vec{r}$ is derived twice with respect to time remembering that $\frac{d \vec{i}}{d t}=\theta \vec{j}$ and $\frac{d \vec{j}}{d t}=-\theta \vec{i}$ acceleration of $m_{b}$ will be

$$
\ddot{\vec{r}}=\left(\ddot{x}-2 \dot{y} \dot{\theta}-y \ddot{\theta}-x \dot{\theta}^{2}\right) \vec{i}+\left(\ddot{y}+2 \dot{x} \dot{\theta}+x \ddot{\theta}-y \dot{\theta}^{2}\right) \vec{j}
$$

Acceleration of $m_{b}$ in the $\vec{j}$ direction will be

$$
\ddot{y}+2 \dot{x} \dot{\theta}+x \ddot{\theta}-y \dot{\theta}^{2}
$$

Longitudinal vibration is ignored, then $\dot{x}=0$, also nonlinear term $y \dot{\theta}^{2}$ is neglected. Inertial load on the unit mass $m_{b}$ of the flexible beam will be 


$$
p(x)=-m_{b}(\ddot{y}+x \ddot{\theta})
$$

When this inertial load is used and $E I$ is assumed constant then EulerBernoulli equation of the beam will be

$$
E I \frac{d^{4} y}{d x^{4}}=-m_{b}(\ddot{y}+x \ddot{\theta})
$$

The governing equation of the motion will be

$$
E I y^{i v}+m_{b} \ddot{y}=-m_{b} x \ddot{\theta}
$$

The mode summation method is assumed for the solution which is

$$
y(x, t)=\sum_{i=1}^{n} \phi_{i}(x) q_{i}(t)
$$

If the orthogonality condition is used and viscous damping is assumed, equation for the generalized coordinate $q_{i}$ is ${ }^{[13,15]}$

$$
\ddot{q}_{i}+2 \zeta \omega_{i} \dot{q}_{i}+\omega_{i}^{2} q_{i}=\frac{\Gamma_{i}}{M_{i}} \ddot{\theta}(t)
$$

Here $\Gamma_{i}$ is defined as mode participation factor which is

$$
\Gamma_{i}=-m_{b} \int_{0}^{l} x \phi_{i}(x) d x
$$

$\mathrm{M}_{\mathrm{i}}$ is defined as generalized mass which is

$$
M_{i}=m_{b} \int_{0}^{l} \phi_{i}^{2}(x) d x
$$

State space form of the differential Equation (8) can be given as

$$
\left[\begin{array}{l}
\dot{x}_{1} \\
\dot{x}_{2}
\end{array}\right]=\left[\begin{array}{cc}
0 & 1 \\
-\omega_{i}^{2} & -2 \zeta \omega_{i}
\end{array}\right]\left[\begin{array}{l}
x_{1} \\
x_{2}
\end{array}\right]=\left[\begin{array}{c}
0 \\
\Gamma_{i} / M_{i}
\end{array}\right] \ddot{\theta}(t)
$$

In the simulations only first orthogonal mode is considered. 


\subsection{Servomotor Motion}

Flexible beam is rotated by a servomotor. Velocity profile is assumed as triangular which is also called bang-bang trajectory. Triangular velocity profile can be given as

$$
\begin{gathered}
\dot{\theta}(t)=\frac{2 \dot{\theta}_{\max }}{t_{r}} t \quad 0 \leq t \leq \frac{t_{r}}{2} \\
\dot{\theta}(t)=2 \dot{\theta}_{\max }-\frac{2 \dot{\theta}_{\max }}{t_{r}} t \quad \frac{t_{r}}{2} \leq t \leq t_{r}
\end{gathered}
$$

Here $\dot{\theta}_{\max }$ is the maximum angular velocity and $t_{r}$ is the rise time. If rise time $t_{r}$ and rotation angle $\theta$ is given, angular velocity and angular acceleration of the rotation can be calculated as

$$
\begin{gathered}
\dot{\theta}_{\max }=\frac{2 \theta_{\max }}{t_{r}} \\
\ddot{\theta}_{\max }=\frac{2 \dot{\theta}_{\max }}{t_{r}} \quad 0 \leq t \leq \frac{t_{r}}{2} \\
\ddot{\theta}_{\max }=-\frac{2 \dot{\theta}_{\max }}{t_{r}} \quad \frac{t_{r}}{2} \leq t \leq t_{r}
\end{gathered}
$$

\subsection{Analytic Solution}

Angular acceleration of the beam rotation for triangular velocity profile is a rectangular wave which can be approximated by a Fourier series as

$$
\ddot{\theta}(t)=\ddot{\theta}_{\max } \frac{4}{\pi}\left[\sin \omega_{r} t+\frac{1}{3} \sin 3 \omega_{r} t+\frac{1}{5} \sin 5 \omega_{r} t+\cdots\right]
$$

Only first three terms are used. $\omega_{r}=\frac{2 \pi}{t_{r}}$ is the fundamental frequency of the Fourier series which is also the rise frequency of the beam. Equation (8) can be written again as

$$
\ddot{q}_{i}+2 \zeta \omega_{i} \dot{q}_{i}+\omega_{i}^{2} q_{i}=\frac{\Gamma_{i}}{M_{i}}\left(\ddot{\theta}_{\max } \frac{4}{\pi}\right)\left[\sin \omega_{r} t+\frac{1}{3} \sin 3 \omega_{r} t+\frac{1}{5} \sin 5 \omega_{r} t+\cdots\right]
$$


Solution of the equation (18) is

$$
q_{i}=X_{0} e^{-\zeta \omega_{n} t} \sin \left(\omega_{d i} t+\phi_{0}\right)+\sum_{n=1,3,5} A_{n} \sin \left(\omega_{n} t-\phi_{n}\right)
$$

$\omega_{d i}=\omega_{i} \sqrt{1-\zeta^{2}}$ is the damped natural frequency of the beam and $\omega_{n}=n \omega_{r}$. Using initial conditions of $q_{i}(0)=0$ and $\dot{q}_{i}(0)=0, \mathrm{X}_{0}$ and $\phi_{0}$ will be obtained as

$$
\begin{gathered}
\phi_{0}=\tan ^{-1} \frac{\sum_{n=1,3,5} A_{n} \sin \phi_{n}}{\frac{\zeta \omega_{i}}{\omega_{d i}} \sum_{n=1,3,5} A_{n} \sin \phi_{n}-\frac{1}{\omega_{d i}} \sum_{n=1,3,5} A_{n} \omega_{n} \cos \phi_{n}} \\
X_{0}=\sqrt{\left(\sum_{n=1,3,5} A_{n} \sin \phi_{n}\right)^{2}+\left(\frac{\zeta \omega_{i}}{\omega_{d i}} \sum_{n=1,3,5} A_{n} \sin \phi_{n}-\frac{1}{\omega_{d i}} \sum_{n=1}^{3} A_{n} \omega_{n} \cos \phi_{n}\right)^{2}}
\end{gathered}
$$

Here

$$
\begin{gathered}
A_{n}=\frac{\frac{A_{i} m}{n \omega_{i}^{2}}}{\sqrt{(m-n)^{2}+(2 \zeta m n)^{2}}} \\
\phi_{n}=\tan ^{-1} \frac{2 \zeta m n}{(m-n)^{2}} \\
t_{r}=m T=m \frac{2 \pi}{\omega_{i}} \\
A_{i}=-\frac{\Gamma_{i}}{M_{i}} \frac{4}{\pi} \ddot{\theta}_{\max }
\end{gathered}
$$

\subsection{Simulation}

For the simulation, Equation (11) is solved by using MATLAB ODE45 ordinary differential equation solver. Steel beam is used. Properties of the steel beam are; elasticity modulus E=207 $\mathrm{Pa}$, mass density $\rho=7700 \mathrm{~kg} / \mathrm{m}^{3}$, length $l=40 \mathrm{~cm}$, width $b=24 \mathrm{~mm}$, thickness $h=$ $0.6 \mathrm{~mm}$. Figures 2 and 3 show the numerical solution of the equation (10) for rise time $t_{r}=2.2 T$ and $t_{r}=3 T$, respectively. The period of the beam for the first natural frequency is $T=0.32 \mathrm{~s}\left(\omega_{n}=19.73 \mathrm{~Hz}\right)$. 
Damping ratio is assumed as $\zeta=0.02$. During the rotation, beam was moving under inertial load, when the rotation stops, the deflection of the beam (at that moment) became an initial displacement for the beam's residual vibration. From the Fig. 2, for $t_{r}=2.2 T=0.70 \mathrm{~s}$, beam tip displacement is very small that is why residual vibration amplitudes of the beam are small. In Fig. 3, rise time is $3 \mathrm{~T}$ which is $0.96 \mathrm{~s}$. Beam deflection at $0.96 \mathrm{~s}$ was bigger than the one for $2.2 \mathrm{~T}$ that is why residual vibration amplitudes were high. Figures 4 and 5 show beam vibration during the rotation of the beam for $t_{r}=2.2 T$ and $t_{r}=3 T$, respectively. Solid line is for analytical solution, dashed line is for numerical solution, which is given in Equation (18). Analytic solution, which uses three term Fourier series expansion of the acceleration of the beam predicts well the motion of the beam. Depending on the rise time, beam tip amplitude at $t=t_{r}$ is changing. Figure 6 shows this change. Values are scaled with respect to the amplitude at $m=1$. At $m=1,3$, and 5 maximum residual vibration amplitudes were making peaks. Between $1.5<m<2.5$ maximum vibration amplitude values were less than $3 \%$ of the amplitude for $m=1$. Between $3.5<m<4.5$ residual vibration amplitudes were less than $0.1 \%$ of the maximum vibration amplitude for $m=1$. These values are independent of the beam natural frequency, that is why these results will not change for different beams.

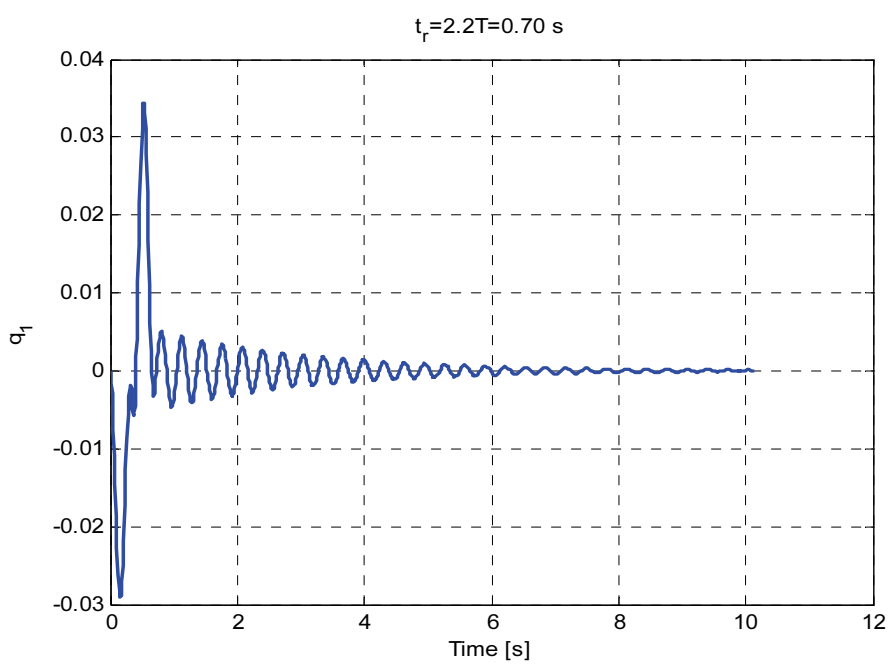

Fig. 2. Rotating beam vibration for $t_{r}=2.2 T$. 


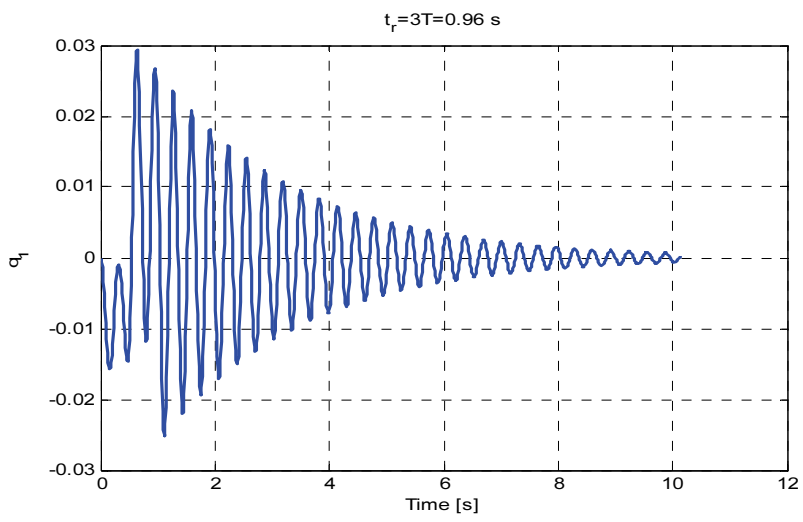

Fig. 3. Rotating beam vibration for $t_{r}=3 T$.

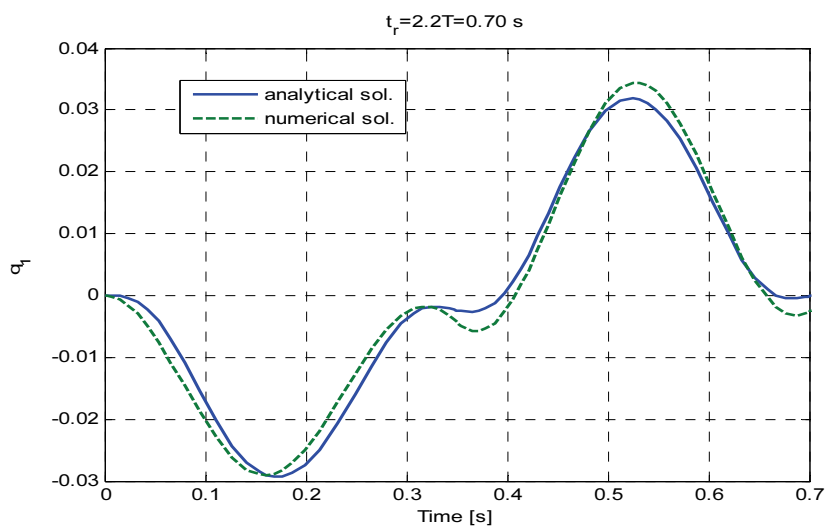

Fig. 4. Rotational motion of the beam during rise time, $t_{r}=2.2 T$.

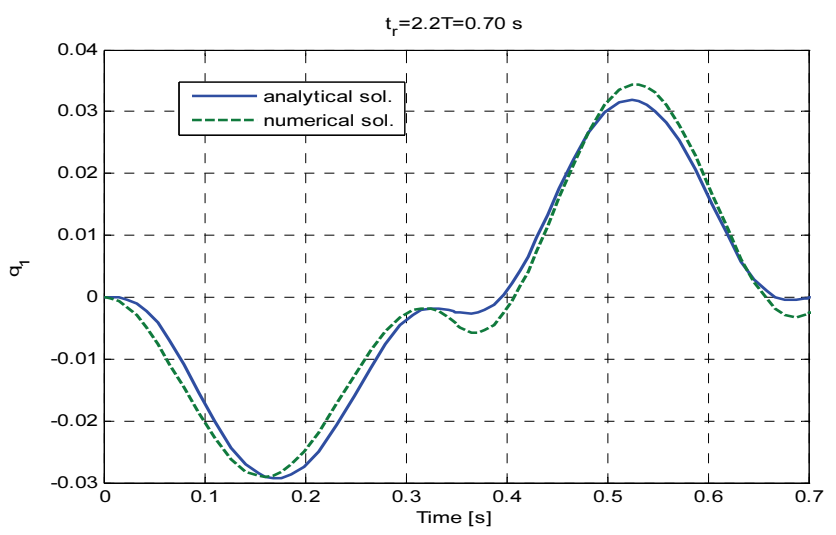

Fig. 5. Rotational motion of the beam during rise time, $t_{r}=3 T$. 


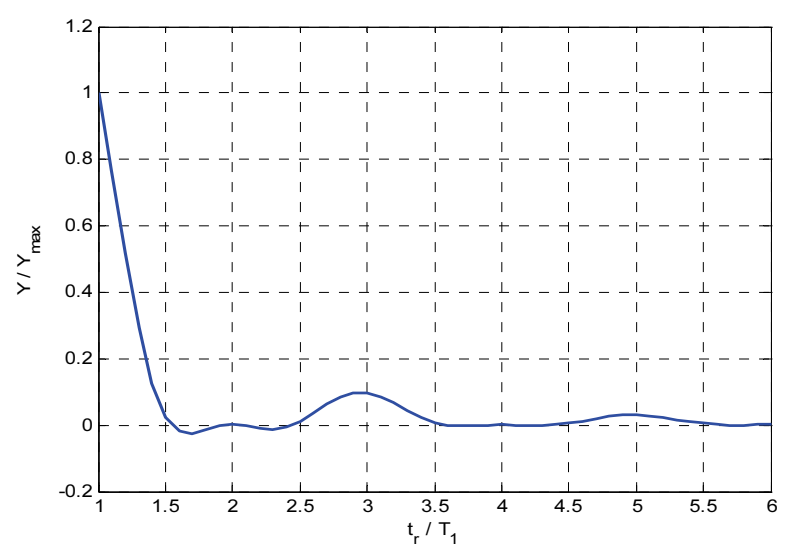

Fig. 6. Residual vibration maximum amplitude spectrum.

\section{Conclusion}

In this study rotating flexible beam equations are derived. Assuming triangular velocity profile for the rotation, analytical and numerical solutions are obtained. Rotational acceleration of the beam, which is a square wave, is approximated by three term Fourier series expansion. Residual vibration maximum amplitude spectrum shows that for the ratio of rise time to beam first natural period values of $1,3,5, \ldots$ maximum residual vibration amplitudes are maximized, for ratio values of 1.5 to 2.5 , maximum residual vibration amplitudes are less than $3 \%$ of the value obtained for ratio $=1$. For ratio values of 3.5 to 4.5 maximum residual vibration amplitudes are less than $0.1 \%$ of the value obtained for ratio $=$ 1. This study shows that it is possible to minimize the residual vibration of the rotating flexible beam by selecting proper rise time.

\section{Acknowledgments}

This research is supported by the Deanship of Scientific Research of the King Abdulaziz University under the grand number of 110/427.

\section{References}

[1] Bhat, S.P., Tanaka, M. and Miu, D.K., Experiments on point to point position control of a flexible beam using Laplace transform technique, Part I: Open Loop, ASME Journal of Dynamic Systems, Measurements and Control, 113: 432-437(1991).

[2] Bhat, S.P. and Miu, D.K., Experiments on point to point position control of a flexible beam using Laplace transform technique, Part II: Closed Loop, ASME Journal of Dynamic Systems, Measurements and Control, 113: 438-443 (1991). 
[3] White, M.W. and Heppler, G.R., Vibration of a rotating Timoshenko beam, ASME Journal of Vibration and Acoustics, 118: 607-613(1996).

[4] Luo, Z.H. and Guo. B.Z., Shear force feedback control of single link flexible robot with a revolute joint, IEEE Transaction on Automatic Control, 42: 53-65(1997).

[5] Low, K.H. and Lau, M.W.S., Experimental investigation of the boundary condition of slewing beam using high speed camera systems, Mechanisms and Machine Theory, 30: 629-643 (1995).

[6] Choi, S.B., Thomson, B.S. and Gandhi, M.V., Control of a single link flexible manipulator fabricated from composite laminates, Journal of Robotic Systems, 12: 45-54 (1995).

[7] Hoa, S.V., Vibration of a rotating beam with tip mass, Journal of Sound and Vibration, 67: 369-381 (1979).

[8] Kojima, H., Vibration of a beam-mass system fixed to a rotating body, Journal of Sound and Vibration, 107: 149-154 (1986).

[9] Mitchell, T.P. and Bruch, J. C., Dynamics of a rotationally accelerated beam, AIAA Journal of Guidance and Control, 12: 935-938 (1989).

[10] Chait, Y., Miklavcic, M. and Maccluer, C.R., Natural modal expansion for the flexible robot arm via self-adjoin formulation, IEEE Transactions on Robotics and Automation, 6 : 601-603 (1990).

[11] Garcia, E. and Inman, D.C., Modeling of a slewing control of a flexible structure, AIAA Journal of Guidance and Control, 14: 736-742 (1991).

[12] Tomei, P. and Tornambe, A., Approximate modeling of robots having elastic links, IEEE Transactions on Systems, Man and Cybernetics, 18: 831-840 (1998).

[13] Ankarali, A. and Diken, H., Vibration control of an elastic manipulator link, Journal of Sound and Vibration, 204: 162-170 (1997).

[14] Shina, K. and Brennan, M.J., Two simple methods to suppress the residual vibrations of a translating or rotating flexible cantilever beam, Journal of Sound and Vibration, 312: 140150 (2008).

[15] Thomson, W.T., Theory of Vibration with Applications, 2nd Ed., Prentice Hall (1981).

[16] Vaughan, J., Yano, A. and Singhose, W., Comparison of Robust Input Shapers, Journal of Sound and Vibration, 315: 797-815 (2008).

[17] Sorensen, K.L. and Singhose, W.E., Command Induced Vibration Analysis Using Input Shaping Principles, Automatica, 44: 2392-2397 (2008). 


\section{الاهتز از ات المتبقية لعمود مرن مقود بمحرك السيرفو}

خالد عبدالله النفيعي، حمزة صلاح الاين دايكن، و عبد الملك علي الغامدي قسم هندسة الإنتاج ، كلية الهندسة ، جامعة الملك عبدالعزبز جدة - المدلكة العربية السعودية

المستخلص: تم في هذه الورقة در اسة الاهنز ازات المتنقية لعهــود

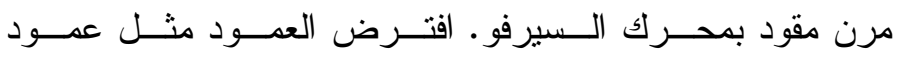

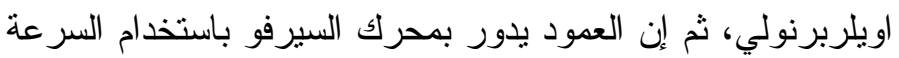

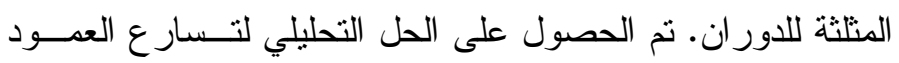
الدور اني باستخدام متسلسلة فورير ـ قمم الاهتزاز ات المتبقية تعتمد

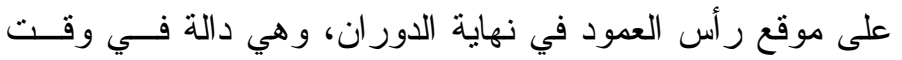

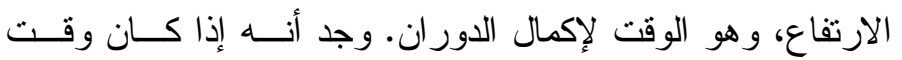

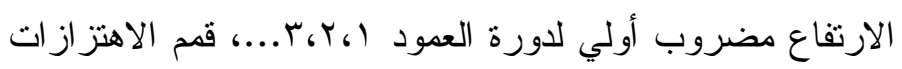
المتبقية نزداد بشكل كبير .

طيف قمم الاهتز ازات المتبقية يبين أنه لوقت الارتفاع لنسب

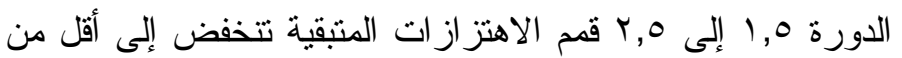

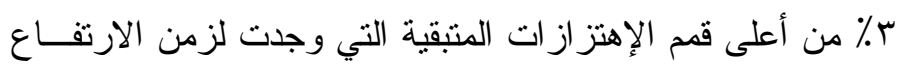
المساوي للدورة الطبيعية الأولى للعمود. 